\title{
The Clinical Study on Chinese Medicine Treatment of Ulcerative Colitis
}

\author{
Ning Zhang 1, 2, a , Zongyue Gao ${ }^{1,2, b}$, Yuyan Liu ${ }^{1,2, c}$ and Xiaoli Zhou ${ }^{3, d^{*}}$ \\ ${ }^{1}$ Henan Province Hospital of TCM, 450000, Henan, P. R. China \\ ${ }^{2}$ The Second Affiliated Hospital of Henan University of Traditional Chinese Medicine, 450000, Henan, \\ P. R. China \\ ${ }^{3}$ The Third Affiliated Hospital of Henan University of Traditional Chinese Medicine, 450000, Henan, P. \\ R. China \\ alantian03zhangning@126.com, 'bnszyygzy@126.com, chnszyylyy@126.com, ${ }^{d}$ sfyzxl@126.com \\ ${ }^{*}$ The corresponding author
}

Keywords: Ulcerative colitis; Chinese medicine treatment; Clinical treatment

\begin{abstract}
Ulcerative colitis is an unknown etiology and recurrence rate of a chronic inflammatory bowel disease. Chinese medicine treatment of ulcerative colitis clinical outcome, side effects, unique approach. Progress article ulcerative colitis pathogenesis, treatment and other aspects from the start, for the clinical treatment of ulcerative colitis provide a reference.
\end{abstract}

\section{Introduction}

Ulcerative colitis (UC) is a kind of mucosa and submucosa infiltration idiopathic nonspecific inflammatory bowel disease in the large intestine, abdominal pain, diarrhea, mucus-like pus and blood, tenesmus as the main symptoms. The disease is more common in Western countries in recent years, UC cases reported from China increased significantly. As the disease etiology and pathogenesis is not clear, and difficult to cure, easy to relapse, it has been the World Health Organization as modern difficult to treat. UC pathogenesis complex, the incidence associated with a variety of factors, but more and more scholars agree $\mathrm{UC}$ is an autoimmune disease, and immunological factors that is most closely related. UC belongs to Chinese medicine, "diarrhea" "dysentery" "bloody" and other areas, including its pathogenesis is virtual and real evil aspects. There are virtual spleen qi deficiency, spleen and kidney deficiency, etc., there is real evil dampness, blood stasis and so on.

\section{Etiology and Pathogenesis of Ulcerative Colitis}

Currently Chinese medicine believes that the cause of ulcerative colitis and more responsibility is to the Congenital deficiency, spleen and stomach, loss of health, when exogenous evil on this basis, internal injuries diet and disease, the pathogenesis of major resistance to evil colon, conduction loss of the Secretary, blood Yongzhi, fat envelope injured stagnation under sepsis. Its pathogenesis is mainly blood and viscera of yin and yang imbalance, loss of transportation usually spleen, intestinal organs stolen Division; or due to liver committed Pitu, kidney is not solid, Turkey dystrophy, transportation of stolen Division; which is spleen disease machine fundamental [1]. There pathological nature of cold, heat points, pathological factors including humidity, heat. Disease location involve the liver, spleen, kidney and colon. Its etiology can be divided into:

Exogenous Heat. Ulcerative colitis patients more in the summer and the onset of the season, when the weather is hot, easy to breed bacteria, food was vulnerable to pollution. Foreign studies have shown that Salmonella, Campylobacter jejuni, Escherichia coli and other bacterial infections are the risk factors for the onset of UC, and anti-infective therapy can effectively relieve the symptoms of UC. Summer and autumn, Yan Shu popular, hot and humid in season, spleen and stomach dull, damp consistency, cement obscure, the culprit stomach, Yu steaming hot and humid, causing spleen and stomach disorders, colon conduction loss of the Secretary, blood block, toxic heat dampness, pump 
knot in the large intestine, the meat rot into pus and disease. After exogenous as dysentery, or lost in the table, or use the law to suppress cold containment evils, loss of government mistreatment; Gao Liang to help them eat early or evil heat, or fill astringent too early, resulting in stomach volt evil, righteousness Haoshang, caused the disease hard to be cured.

Improper Diet. In clinical studies found, UC onset or recurrence of taste and eating acrid, such as chives, parsley, fennel, onions, garlic and other spices divergence of the goods, cold or unclean food, and eating Hunxing especially beef and mutton, fish seafood related. Drinking and eating disorders can also induce or aggravate the disease. Ulcerative colitis, a disease, the main cause of stagnation in the intestinal organs cult poison blood lipid membranes, combined with the heat of the drug fumigation and hair. Eating spicy and spicy take channeling the product is easy to Haoshang human blood, eating beef and mutton and fish and seafood and other food will increase the gastrointestinal toxins, so hot and humid Fuxie deficiencies, fouling is not eliminated, complex gas injury consumption of blood, stagnation in the arrest and aggravated clinical often abdominal pain, increased stool mucus pus and blood, thick greasy yellow tongue and other symptoms.

Emotional Disorders. Common clinical UC patients due Concerned angry and other emotional changes caused by exacerbations or recurrence. Modern studies have shown that psychological stress is closely related to disease activity of UC, UC constitutes a patient susceptible to personality defects and internal quality based UC disease. Emotional disorders caused by UC, primarily related to the liver, spleen, lungs. The liver is anger in the Chi, angry can make the liver hyperactivity or stagnation, caused by the transportation of coke unfavorable, satisfied by the Division lost ${ }^{[2]}$. Valley stop is stagnant, water retention wet, lack of heat, stagnation Damp colon, bowel gas barrier, intestinal collaterals stasis, blood lost flesh rot and see abdominal pain, lower interest sepsis. Spleen in the Chi thinking, long-term mental stress, depression may be thinking injured spleen, spleen caused by transport and adverse, wet stop stagnation, off in the intestine, large intestine conduction loss of the Secretary, may cause blood pump end, hurt the blood lipid membrane envelope, rot into pus and the incidence or recurrence. In the annals of lung sad, or overwhelmed by grief, or because Suoyu fail may cause lung canopy, the air-lift disorders, colorectal conduction loss of the Secretary, within the stagnation of Health, which ultimately caused the heat, blood stasis mutual knot of the hair disease.

Overworked. UC lingering disease, chronic illness stomach injury, caused by receiving no right, Kin Yun dereliction of duty. Need to carefully nursed back to health in daily life, so the spleen and stomach was healthy, moisture was based, was eliminate toxins, blood reconcile, in order to prevent the recurrence of the disease. Common clinical patient because of work, irregular work and rest or fatigue life caused by disease or relapse. Overstrain the stomach is more true, ingestion Needless, voicing, implanted with Fuxie fouling the gut, then see loose stools thin, increased mucus, and even the urgency watery diarrhea; temper weakness, blood biochemistry lack of sources, not on the wing in the face to meet the color dark complexion; not filling up the limb, muscle, so see physical fatigue, lassitude; disease for a long Spleen damage, have no right to transport, drinking water, stay in the intestine were seen Luk Luk bowel; yin yang, cold from birth, so see aversion to cold abdomen. Total due Fuxie fouling is not removed, blood loss, combined with the coke damage caused disease recurrence or delayed healing.

Doctors believe genetic factors, immune factors, intestinal infection factors, apoptosis, environmental factors, psychological factors and so on.

Immune Factors. Currently modern medicine for the pathogenesis of ulcerative colitis is not very clear, the most studied is the most detailed aspects of immunity, the immune disorder is considered a key link. In the colonic mucosa of patients with UC can be detected in a large number of lymphocytes, plasma cells, macrophages, neutrophils exist, these immune cells can release various cytokines. Cytokines as intercellular signaling molecules, target specific receptors on the cell membrane, plays an important role in the immune response and inflammatory reaction in the body. Cytokines (cytokine) mainly refers to certain non-immune cells and immune cells to synthesize a class of small secreted molecules having a wide range of biologically active peptide substances. According to substantially the role of cytokines, cytokine often divided into three categories: pro-inflammatory cytokines, 
inflammatory cytokines, and growth factors. Proinflammatory cytokines include interleukin -1 (IL-1), IL-2, IL-6, IL-8, IL-12, tumor necrosis factor $-\alpha$ (TNF- $\alpha)$, TNF- $\beta$, IFN, etc., most of cytokine produced by monocytes and macrophages, involved in cell-mediated immune response. Anti-inflammatory cytokines include IL-4, IL-5, IL-10, IL-13 and the like. Produced mainly by T cells involved in the humoral immune response. Another class of growth factors include growth-stimulating factor, transforming growth factor (TGF), epidermal growth factor (EGF), insulin-like growth factor, fibroblast growth factor, cells have different growth regulators and proliferation. Imbalance of proinflammatory cytokines and anti-inflammatory cytokines were considered as an important in the pathogenesis of UC.

Genetic Factors. The incidence of ulcerative colitis may be related to genetic predisposition. The results show that some scholars, high NAT1, BPI and CXCL9 in UC group expression levels, the difference was statistically significant $(\mathrm{P}<0.05)$, consistent with the microarray results, HLA-DQB1 compared with the normal group upregulated in UC group, the difference was statistically significant ( $\mathrm{P}<0.05)$, with the microarray results do not coincide. BPI, CXCL9, NAT1 and HLA-DQB1 expression and gender, age and severity unrelated gene (P>0.05), showed that patients with UC BPI showed high expression, CXCL9 in UC patients with high expression of inflammatory cytokines and anti-inflammatory cytokines related imbalance between. Expression of HLA-DQB1 abnormalities in patients with UC also further illustrates the importance of genetic factors in the pathogenesis of UC. BPI, consistent NAT1, CXCL9 in UC patients and the expression of the gene chip results proved that the relative reliability of gene chips. The test results with the results of HLA-DQB1 gene chip does not coincide with a variety of possible factors, such as genetic polymorphism, a small number of samples, different people, different inter-ethnic differences.

\section{Clinical Treatment}

Chinese Medicine Treatment. Ni Hai [3] will be divided into inner heat ulcerative colitis, type spleen and stomach, spleen and kidney yang, liver and spleen deficiency, blood stasis type five types. Damp type of medicinal decoction Pulsatilla (Pulsatilla $15 \mathrm{~g}$, fried berberine $10 \mathrm{~g}$, ash bark $10 \mathrm{~g}$, white peony root $15 \mathrm{~g}$, licorice $5 \mathrm{~g}$, nutmeg $10 \mathrm{~g}$, Burnet $15 \mathrm{~g}$, wood $10 \mathrm{~g}$, nosed pit viper tongue grass $20 \mathrm{~g}$ ). Spleen and stomach type of medicinal SHENLINGBAISHU Powder (Codonopsis $15 \mathrm{~g}$, Yam $12 \mathrm{~g}$, lotus seeds and meat $12 \mathrm{~g}$, Atractylodes $15 \mathrm{~g}$, Poria $10 \mathrm{~g}$, Coix $10 \mathrm{~g}$, Amomum $10 \mathrm{~g}$, lentils $10 \mathrm{~g}$, licorice $8 \mathrm{~g}$ ). Spleen and kidney yang medicinal Sishen Pill (psoralen $10 \mathrm{~g}$, Evodia $10 \mathrm{~g}$, nutmeg $15 \mathrm{~g}$, Schisandra $15 \mathrm{~g}$, Codonopsis $15 \mathrm{~g}$, Atractylodes $10 \mathrm{~g}$, licorice $6 \mathrm{~g}$, ginger $6 \mathrm{~g}$ ). Liver and spleen deficiency medicinal Tongxieyaofang Decoction (Citrus $10 \mathrm{~g}$, Atractylodes $10 \mathrm{~g}$, white peony root 15 $\mathrm{g}$, wind $15 \mathrm{~g}$, Bupleurum $10 \mathrm{~g}$, Pinellia $10 \mathrm{~g}$, licorice $5 \mathrm{~g}$ ). Qi stagnation type of medicinal Gexiazhuyutang subtraction (Wulingzhi $10 \mathrm{~g}$, angelica $15 \mathrm{~g}$, Chuanxiong 10g, peach kernel $15 \mathrm{~g}$, Dan $8 \mathrm{~g}$, red peony root $10 \mathrm{~g}$, Lindera $10 \mathrm{~g}, 10 \mathrm{~g}$ Yuan Hu, sweet $10 \mathrm{~g}$, safflower $5 \mathrm{~g}$, Magnolia $20 \mathrm{~g}$, licorice $5 \mathrm{~g}$ ). Results 58 patients were cured 31 cases (53.4\%), 17 cases $(29.3 \%)$, improved in 8 cases $(13.8 \%), 2$ cases $(3.4 \%)$.

Enema. Li Wei [4]with SHENLINGBAISHU Powder (Codonopsis $25 \mathrm{~g}$, Atractylodes $20 \mathrm{~g}$, Yam $15 \mathrm{~g}$, Astragalus $30 \mathrm{~g}$, Pulsatilla $20 \mathrm{~g}$, Treats $20 \mathrm{~g}$, berberine $10 \mathrm{~g}$, ash bark $10 \mathrm{~g}$, dried tangerine peel $12 \mathrm{~g}$, wind $15 \mathrm{~g}$, white peony root $10 \mathrm{~g}$, licorice $10 \mathrm{~g}$ ) enema for ulcerative colitis 35 cases a day, concentrated fry $150 \mathrm{~mL}$, bedtime enema, 15 days for a course of treatment with 1 to 3 treatment, results: 17 cases were cured, 11 cases markedly effective in 5 cases, 2 cases, the total effective rate was $94.3 \%$.

Chinese Herbs Addition Enema. Wangmei Rong [5] using Pulsatilla Decoction Liujunzi Tang CD orally (Pulsatilla, Qin skin, berberine, Codonopsis, Atractylodes, Poria, Citrus, Pinellia, Citrus aurantium, peony, hawthorn, Coix seed, woody, angelica, red flowers, licorice) orally, at the same time to the prescription (Sophora, Cork, Burnet, gall, white, and, seven powder) retention enema treatment UC38 cases, the total efficiency of $100 \%$, significantly better than the control group. 
Other Therapies: In addition to the commonly used method of oral medicine, Chinese medicine enema, the Chinese medicine treatment of ulcerative colitis, there are many other flexible, small damage to the body treatments, such as massage, point application, massage, acupuncture, cupping, injection, moxibustion, scraping, embedding and other less investment, low cost, effective and easy for patients to accept.

\section{Summary}

Chinese medicine treatment of ulcerative colitis clinical efficacy is better, especially in stable disease, relapse prevention has obvious advantages. Spleen and stomach, transport and loss of health is the pathological basis of the disease, Damp intestine is the major pathological factors, blood lipid membrane envelope damaged intestine, blood lost flesh rot into ulcers is the main pathogenesis, which is the basic pathogenesis of this disease law, but also constitute the main basis for clinical use. That is to help transport the spleen, bowel dampness, cooling blood Ning network, clear and warm with the basic tone of this disease phlegm, myogenic convergence of ulcers and other treatment. Clinical medicine should be emphasized Thanh Hoa heat, consistent; relevant organs, overall modulating; Regulating blood, caution Guse principles. Through diagnosis and treatment, with the addition and subtraction cards to meet the needs of individualized treatment, the use of Chinese medicine treatment characteristics advantages for the majority of patients to redress grievances. Chinese medicine treatment of ulcerative colitis using a method more effective, the use of flexible, less adverse reactions. Chinese medicine emphasizes the integrity of people, in the treatment of ulcerative colitis patients pay more attention to subjective feelings, more individualized in efficacy evaluation, more accurate, more objective and more comprehensive, but in syndrome classification, TCM Differential Treatment, there are studies on the selection and sample size, and evaluate the efficacy of the control group some problems, mechanism of action of various treatments requires further exploration and clear, its efficacy remains to be further improved.

\section{References}

[1] Wang Rui. TCM Comprehensive Treatment of ulcerative colitis in 30 cases [J] Hubei traditional Chinese Medicine, 2005, 27 (12): 23-24.

[2] Zhao Shuping. Advances in medicine in the treatment of ulcerative colitis [J] Advances in Clinical Study on, 2004, 21 (6): 6-7.

[3] Ni Hai.Diagnosis and treatment of ulcerative colitis 58 cases [C] Gansu Province Medical Association 2010 Annual Conference and member representative Paper Series, 2010: 249-250.

[4] Li Wei, Zhao Hong, Tang Zhi. Clinical observation of 35 cases [J] enema treatment of ulcerative colitis Jilin Traditional Chinese Medicine, 2006, 26 (2): 29.

[5] Wangmei Rong, Yin Jin, Peng Xiaojun. Colitis Clinical internal medicine and enema treatment of ulcerative [J] Journal of Hubei TCM, 2007, 29 (9): 38. 